\title{
Scale-free patterns at a saddle-node bifurcation in a stochastic system
}

\author{
Mami Iwata* and Shin-ichi Sasa ${ }^{\dagger}$ \\ Department of Pure and Applied Sciences, University of Tokyo, \\ 3-8-1 Komaba Meguro-ku, Tokyo 153-8902, Japan \\ (Dated: Received 6 June 2008; published 14 November 2008)
}

\begin{abstract}
We demonstrate that scale-free patterns are observed in a spatially extended stochastic system whose deterministic part undergoes a saddle-node bifurcation. Remarkably, the scale-free patterns appear only at a particular time in relaxation processes from a spatially homogeneous initial condition. We characterize the scale-free nature in terms of the spatial configuration of the exiting time from a marginal saddle where the pair annihilation of a saddle and a node occurs at the bifurcation point. Critical exponents associated with the scale-free patterns are determined by numerical experiments.
\end{abstract}

PACS numbers: 82.40.Ck, 82.40.Bj, 05.40.-a, 05.65.+b

Scale-free patterns are widely observed in nature. The most familiar one in statistical mechanics might be that in particle configurations at the gas-liquid critical point [1]. The patterns are characterized by a correlation length which is given by a power-law function of the distance from the critical temperature. Another type of scale-free patterns was found in diffusion-limited aggregation [2] and spinodal decomposition [3]. In this type, the length scale that characterizes the patterns grows as a power-law function of time, without the fine-tuning of the system parameters.

Recently, a new type of scale-free pattern called $d y$ namical heterogeneity has been found in glassy systems $[4,5,6,6,7,8,9,10]$. Such patterns are fascinating because they become visible only by quantifying some dynamical events (called bond-breaking events or unlocking events) during a particular time interval $t_{*}$. Let us consider dense colloidal suspensions as an example. We denote the displacement of particles in a region around a position $x$ during a time interval $t$ as $q(x, t)$. Then, the correlation length $\xi(t)$ of the pattern $q(x, t)$ becomes maximum at a time $t_{*}$ and $\xi\left(t_{*}\right)$ exhibits divergent behavior as a function of the distance from a critical parameter value.

The characterization in terms of the time-dependent correlation length $\xi(t)$ is similar to that of growing patterns, while the existence of the critical parameter value is similar to critical phenomena in equilibrium systems. Such a coexistence of these features implies that $q\left(x, t_{*}\right)$ indeed belongs to a new type of scale-free patterns. Since its nature is simple and nontrivial, we expect that there exists a wide class of systems that exhibit such a type of patterns. Motivated by this expectation, we propose the simplest model among them. We then intend to elucidate the nature of the new type of scale-free patterns.

Model: In the present paper, we study a spatially extended stochastic system that exhibits relaxation behavior at a saddle-node bifurcation. Although there is a

*Electronic address: iwata@jiro.c.u-tokyo.ac.jp

${ }^{\dagger}$ Electronic address: sasa@jiro.c.u-tokyo.ac.jp certain idea that connects this model with the understanding of glassy systems [11, 12], we do not consider the relationship in this paper. Instead, we regard this model as a typical system that undergoes an elementary bifurcation under the influence of noise. By elementary bifurcation, we imply pitch-fork bifurcation, Hopf bifurcation, and saddle-node bifurcation 13]. Among them, the first two bifurcations under the influence of noise have been studied intensively in the the context of critical phenomena [14, 15, 16]. Therefore, a saddle-node bifurcation with noise might be related to a new class of critical phenomena.

Specifically, we study a coupled Langevin equation in a one-dimensional lattice $\{i \mid i=1,2, \cdots, N\}$. Let $\phi_{i}$ be a one-component quantity defined at the $i$-th site, where we assume periodic boundary conditions $\phi_{0}=\phi_{N}$ and $\phi_{N+1}=\phi_{1}$. Then, $\phi_{i}$ obeys

$$
\partial_{t} \phi_{i}=f\left(\phi_{i}\right)+\kappa\left(\phi_{i+1}+\phi_{i-1}-2 \phi_{i}\right)+\xi_{i},
$$

where $f(\phi)=-\phi\left((\phi-1)^{2}+\epsilon\right)$ with small $\epsilon ; \kappa$ is a coupling constant and $\xi_{i}$ represents Gaussian white noise that satisfies $\left\langle\xi_{i}(t) \xi_{j}\left(t^{\prime}\right)\right\rangle=2 T \delta\left(t-t^{\prime}\right) \delta_{i j}$. The noise intensity $T$ is assumed to be a small positive constant. The potential function $v(\phi)$, which is defined by $f=-\partial_{\phi} v$, has a single minimum at $\phi=0$ when $\epsilon \geq 0$, while a pair comprising the minimum and maximum appears around $\phi=1$ when $\epsilon$ becomes negative (see Fig. 1). The minimum and maximum correspond to the node and saddle point, respectively, in the deterministic equation $\partial_{t} \phi=f(\phi)$. Such a qualitative change in the trajectories at $\epsilon=0$ in this deterministic equation is called saddle-node bifurcation. We call the fixed point $\phi=1$ for $\epsilon=0$ marginal saddle because this saddle vanishes when $\epsilon>0$. A characteristic feature of (1) is that even small noise drastically affects the trajectories passing through the marginal saddle. For example, when $\epsilon \leq 0$, the deterministic trajectories starting from $\phi=1.2$ reach the fixed point near $\phi=1$, while trajectories under the influence of noise escape from this fixed point and finally arrive at the region near the globally minimum point $\phi=0$. In the argument below, in order to extract a simple relation, we focus on the case $\epsilon=0$ at which the saddle-node bifurcation occurs. 


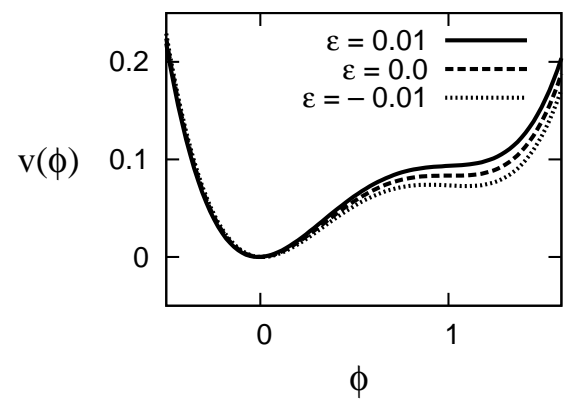

FIG. 1: Potential $v$ as a function of $\phi$ for several values of $\epsilon$.

Since the model we study might be the simplest in the class of spatially extended stochastic systems in which a saddle-node bifurcation occurs locally in space, our model has some relevance to many experimental systems. One example is fluctuating motion of a single one-dimensional polymer that is subjected to a trapping potential in a liquid (see Ref. [17]). Another example might be found in stochastic reaction-diffusion systems because a saddle-node bifurcation is observed in chemical reactions (see Ref. [18] as an illuminating example). Although multiplicative noise generally occurs in chemical reaction systems, we expect that the multiplicative nature does not affect the behavior reported below unless the noise intensity becomes zero at the marginal saddle.

We investigate (11) by numerical simulations. Concretely, we employ an explicit discrete method with a time step $\Delta t=0.01$, where the error is $O\left(\Delta t^{3 / 2}\right)$. We have confirmed that the important results reported below are kept to be valid when we select $\Delta t=0.001$. We also restrict our investigations to the case $\kappa=1$ We obtained the same result for the case $\kappa=0.5$. That is, a fine-tuning of the parameter value is not necessary [19]. Finally, since we are interested in the patterns emerging from a homogeneous state, we assume that $\phi_{i}(0)=1.2$.

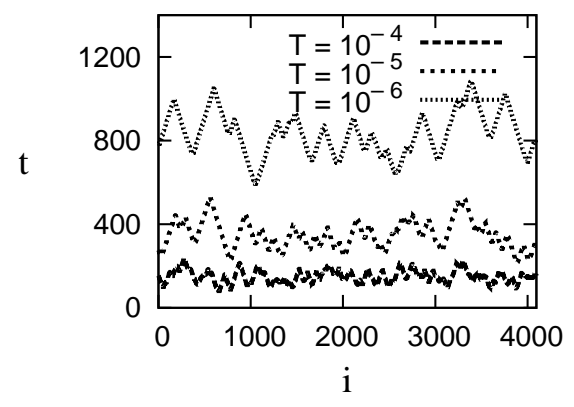

FIG. 2: Contour curves defined by $\phi_{i}(t)=0.5$ for several values of $T . N=4096$.

Preliminaries: In order to observe the time evolution of $\phi_{i}$, we display contour curves defined by $\phi_{i}(t)=0.5$ for several values of $T$ (see Fig. 2). Each contour curve distinguishes the late stage $\left(\phi_{i}(t) \simeq 0\right)$ from the early stage $\left(\phi_{i}(t) \simeq 1\right)$ because the two stages are connected

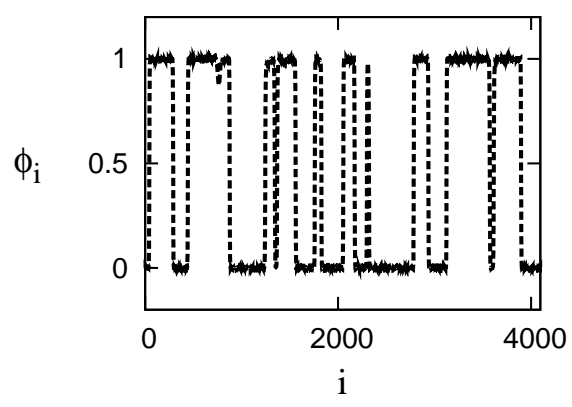

FIG. 3: Spatial pattern at time $t=828$ for the system with $T=10^{-6} . N=4096$.

in a short time interval around the exiting time from the marginal saddle at each site $i$. Thus, for example, the pattern $\phi_{i}$ at time $t=828$ for the system with $T=10^{-6}$ consists of domains $\phi_{i} \simeq 1$ and $\phi_{i} \simeq 0$, as shown in Fig. 3. where the time $t=828$ is selected such that the spatial average of $\phi_{i}$ becomes 0.5 . It should be noted that such a pattern appears only around a particular time $t=t_{*}$. Furthermore, the typical length scale of the pattern at the time $t_{*}$ increases as $T$ is decreases. This suggests that $\phi_{i}\left(t_{*}\right)$ is scale-free in the limit $T \rightarrow 0$.

The behavior of $\phi_{i}(t)$ is similar to that of a field $q(x, t)$ describing a dynamical event in glassy systems (see Ref. [4]). In order to make the analogy more explicit, we consider the spatial average of $\phi_{i}, \bar{\phi}(t)=\sum_{i} \phi_{i}(t) / N$. In Fig. 4. we show the ensemble average $\langle\bar{\phi}(t)\rangle$ for the system with $T=10^{-a}$, where $a=3,4,5$, and 6 . The staying time at the marginal saddle increases as $T$ is decreased. The graph of $\langle\bar{\phi}(t)\rangle$ is similar to a time correlation function of density fluctuations in glassy systems. Furthermore, we measure the fluctuation intensity

$$
\chi_{\phi}(t)=N\left(\left\langle\bar{\phi}^{2}(t)\right\rangle-\langle\bar{\phi}(t)\rangle^{2}\right) .
$$

As shown in Fig. 5. $\chi_{\phi}(t)$ has one peak at a time $t=t_{*}$, which provides a precise definition of $t_{*}$ intuitively used in the previous paragraph. Then, $\chi_{\phi}\left(t_{*}\right)$ increases as $T$ is decreased. In fact, the inset of Fig. 5 suggests that $\chi_{\phi}\left(t_{*}\right)$ exhibits the power-law divergence

$$
\chi_{\phi}\left(t_{*}\right) \simeq T^{-\gamma_{\phi}},
$$

where the value of the exponent $\gamma_{\phi}$ is close to $1 / 3$. This divergent behavior corresponds to the dynamical heterogeneity in glassy systems. These observations naturally lead us to a conjecture that the correlation length of $\phi_{i}\left(t_{*}\right)$ exhibits the power-law divergence as a function of $T$. In this paper, we do not investigate $\phi_{i}\left(t_{*}\right)$ directly but propose a novel quantity that characterizes the divergent behavior.

Result: Our basic idea for the characterization of the patterns is to employ a special solution $\phi_{*}(t)$ of the equation $\partial_{t} \phi=-\partial_{\phi} v(\phi)$ under the conditions $\phi(t) \rightarrow 1$ for $t \rightarrow-\infty, \phi(t) \rightarrow 0$ for $t \rightarrow \infty$, and $\phi(0)=0.5$. We then express the time evolution of the patterns as

$$
\phi_{i}(t)=\phi_{*}\left(t-\theta_{i}\right)+\rho_{i}\left(t-\theta_{i}\right),
$$




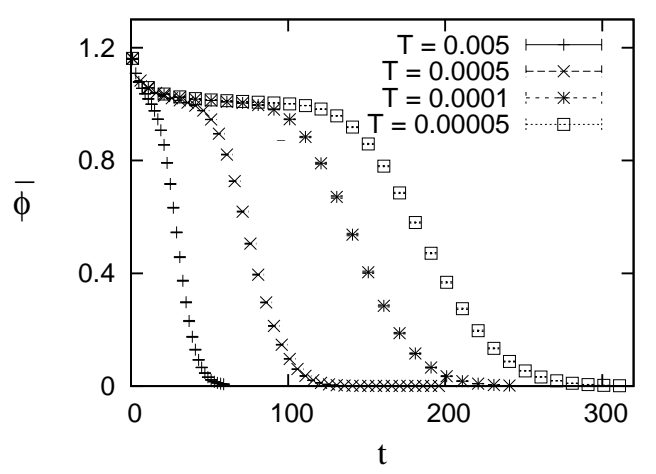

FIG. 4: $\bar{\phi}$ as a function of $t . N=1024$.

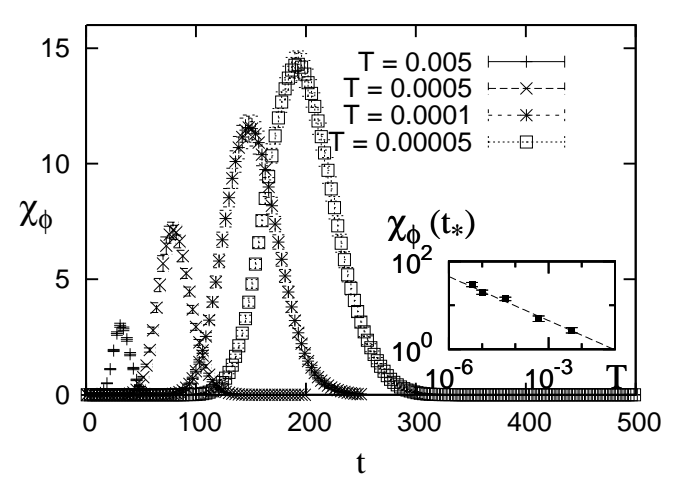

FIG. 5: $\chi_{\phi}$ as a function of $t . N=1024$. Inset: $\chi_{\phi}\left(t_{*}\right)$ as a function of $T$ in a log-log scale. The guide line represents $\chi_{\phi}\left(t_{*}\right) \simeq T^{-1 / 3}$.

where $\theta_{i}$ represents the exiting time from the marginal saddle, which is defined by $\phi_{i}\left(\theta_{i}\right)=0.5$. By this definition, the pattern of $\theta_{i}$ corresponds to the contour curve shown in Fig. 2, For a typical trajectory whose weight is large, $\rho_{i}\left(t-\theta_{i}\right)$ is expected to be smaller than the special solution. Here, we conjecture that the fluctuation intensity of $\theta_{i}$ exhibits a power-law divergence of $T$ because the divergence is related to the Goldstone mode associated with the symmetry breaking of the time translational symmetry. We thus characterize the conjectured scale-free patterns by the statistical quantities of $\theta_{i}$.

Concretely, by introducing the Fourier transform of $\theta_{i}$ as

$$
\tilde{\theta}\left(k_{n}\right) \equiv \frac{1}{N} \sum_{j=1}^{N} \mathrm{e}^{i k_{n} j} \theta_{j}
$$

we measure the spectrum

$$
\chi\left(k_{n} ; T\right) \equiv N\left\langle\left|\tilde{\theta}\left(k_{n}\right)\right|^{2}\right\rangle,
$$

where $k_{n}=2 \pi n / N$ with $n=1,2, \cdots, N / 2$. Here, \langle\rangle represents the ensemble average. In order to extract the singular behavior of $\chi\left(k_{n} ; T\right)$ in the limit $T \rightarrow 0$, we first assume a scaling relation

$$
\chi\left(k_{n} ; T\right)=T^{-\gamma} \tilde{\chi}\left(k_{n} T^{-\nu}\right),
$$

where the exponents $\gamma$ and $\nu$ characterize the divergences of the amplitude of the spectrum $\chi\left(k_{n} ; T\right)$ and its length scale, respectively, in the limit $T \rightarrow 0$. The exponents $\gamma$ and $\nu$ are determined such that $\chi\left(k_{n} ; T\right)$ with $T=10^{-a}$, where $a=3,4,5$, and 6 , collapse into one universal curve. However, after some trials, we find that a logarithmic correction appears in the scaling relation. To demonstrate it, we express $\chi\left(k_{n} ; T\right) T$ as a function of $k_{n} T^{-1 / 3} /(-\ln T)^{1 / 2}$ in Fig. 6. which suggests that the scaling relation (7) with the logarithmic correction appears to be valid for the values

$$
\begin{aligned}
& \gamma=1, \\
& \nu=1 / 3 .
\end{aligned}
$$

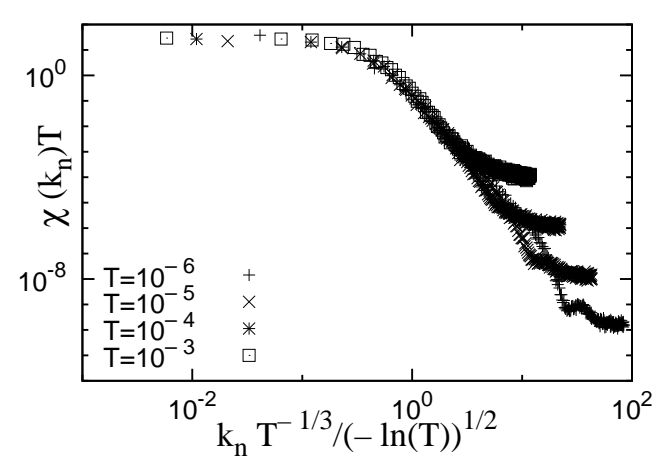

FIG. 6: $\quad \chi\left(k_{n} ; T\right) T$ as a function of $k_{n} T^{-1 / 3} /(-\ln T)^{1 / 2}$ for several values of $T . N=4096$. The statistical error-bar is about the symbol size. By comparing the systems with $N=512,1024,2048$, and 4096 , it is found that the finite-size effects are fairly small.

The exponent $\gamma$ is related to the scale dimension of the quantity $\theta_{i}$. In order to confirm this fact explicitly, we consider the spatial correlation function

$$
\left\langle\theta_{l} \theta_{m}\right\rangle=T^{-(\gamma-\nu)} \frac{\Delta K}{2 \pi} \sum_{n=1}^{N} \mathrm{e}^{-i K_{n}(l-m) T^{\nu}} \bar{\chi}\left(K_{n}\right),(10)
$$

where $K_{n}=k_{n} T^{-\nu}$ and $\Delta K=T^{-\nu} 2 \pi / N$. From (10), we find a scaling form

$$
\theta_{l}=T^{-(\gamma-\nu) / 2} \Theta\left(T^{\nu} l\right),
$$

where $\Theta(x)$ is a fluctuating field whose distribution function is independent of $T$ in the limit $T \rightarrow 0$. By combining (44) with this scaling form (11), we arrive at our main claim that the scale-free patterns are characterized by

$$
\phi_{i}(t) \simeq \phi_{*}\left(t-T^{-1 / 3} \Theta\left(T^{1 / 3} i\right)\right),
$$

which clearly indicates the scale-free nature at a saddlenode bifurcation point in the limit $T \rightarrow 0$. We remark that the statistical quantities of $\phi_{i}$, including the critical exponent $\gamma_{\phi}$ in (3), can be calculated theoretically from our result (12). We believe that the representation (12) captures the essence of the scale-free pattern. 
Concluding remarks: We have proposed a new universality class consisting of stochastic systems undergoing a saddle-node bifurcation. A remarkable feature of this class is that the criticality near a saddle-node bifurcation originates from the fluctuations of the time passing through a marginal saddle. We expect that there is a rich variety of systems belonging to this class, although a quantitative experimental measurement seems to be challenging. An important example includes neuronal avalanches [20], which have recently been studied extensively. The analysis of a simple mean-field model has revealed the critical nature clearly [21]. We conjecture that scale-free patterns similar to those studied in this paper will be observed in systems related to neuronal avalanches.

Furthermore, we have pointed out that the cooperative behavior observed in our model is related to the dynamical heterogeneity in glassy systems. We wish to emphasize that this similarity is not superficial. In fact, recently, we have found that the dynamics of $k$-core percolation in a random graph exhibits a jamming transition via a saddle-node bifurcation of some order parameter in the thermodynamic limit 22].

The most important result is the expression (12), which claims that the criticality of the pattern observed at a particular time is characterized by $\theta_{i}$. The approach based on this idea was addressed in the theoretical analysis of a model [12]. We expect that this approach would elucidate the nature of the criticality of jamming systems.

Before ending this paper, we present some important future problems. The first problem is to understand the behavior of $d$-dimensional systems. Theoretically, we should start estimating the upper-critical dimension by using physical arguments. The second problem is to uncover possible normal forms in multi-component systems. Even if the deterministic part exhibits a saddle-node bifurcation, the effect of noise is not determined uniquely in such systems. There might be some systems in which the exponents are altered from the result we obtained in this paper. Finally, the $\epsilon$-dependence of the length scale should be clarified. These will define another exponent, which might be more relevant in the context of critical phenomena.

In order to consider these future problems, we need to develop the theoretical analysis. We have just finished the mean field analysis, which will be published soon [23]. According to the result, the field $\theta$ scales as $\theta \simeq T^{-1 / 3}$ and there are scaling relations as functions of $\epsilon T^{-2 / 3}$. From the naive dimensional analysis, the length scale of the system with $\epsilon \neq 0$ turns out to be proportional to $\epsilon^{-1 / 2}$, which leads to $\gamma=1$ and $\nu=1 / 3$.

This work was supported by a grant from the Ministry of Education, Science, Sports and Culture of Japan, No. 19540394. Mami Iwata acknowledges the support by Hayashi Memorial Foundation for Female Natural Scientists.
[1] H. E. Stanley, Introduction to Phase Transitions and Critical phenomena (Oxford Univ. Press, Oxford, 1971).

[2] H. J. Herrmann, Physics Reports 136, 153 (1986).

[3] A. J. Bray, Adv. Phys. 43, 357 (1994).

[4] R. Yamamoto and A. Onuki, Phys. Rev. E 58, 3515 (1998).

[5] L. Berthier, G. Biroli, J.-P. Bouchaud, L. Cipelletti, D. El Masri, D. L'Hôte, F. Ladieu, and M. Pierno, Science 310, 1797 (2005).

[6] A. C. Pan, J. P. Garrahan, and D. Chandler, Phys. Rev. E 72, 041106 (2005).

[7] O. Dauchot, G. Marty, and G. Biroli, Phys. Rev. Lett. 95265701 (2005).

[8] E. R. Weeks, J. C. Crocker, A. C. Levitt, A. Schofield, and D. A. Weitz, Science 287, 627 (2000).

[9] G. Biroli and J. P. Bouchaud, Europhys. Lett. 6721 (2004).

[10] G. Biroli, J. P. Bouchaud, K. Miyazaki, and D. R. Reichman, Phys. Rev. Lett. 97, 195701 (2006).

[11] M. Iwata and S. Sasa, J. Stat. Mech. L10003 (2006).

[12] M. Iwata and S. Sasa, Europhys. Lett. 77, 50008 (2007).

[13] J. Guckenheimer and P. Holmes, Nonlinear Oscillations,
Dynamical Systems and Bifurcations of Vector Fields (Springer-Verlag, New York, 1983).

[14] N. Goldenfeld, Lectures on Phase Transitions and the Renormalization Group (Addison-Wesley, New York, 1992).

[15] Y. Kuramoto, Chemical Oscillations, Waves, and Turbulence (Springer, Berlin, 1984).

[16] H. Daido, J. Stat. Phys. 60, 753 (1990).

[17] G. Costantini and F. Marchesoni, Phys. Rev. Lett. 87, 114102 (2001).

[18] J. J. Tyson, K. C. Chen, and B. Novak, Current Opinion in Cell Biology 15, 221 (2003).

[19] However, according to our preliminary theoretical analysis, the system with large $\kappa$ might exhibit different behavior, which will be studied in future.

[20] D. Plenz and T. C. Thiagarajan, Trends in Neurosci. 30, 101 (2007).

[21] H. Ohta and S. Sasa, e-print arXiv:0805.4671

[22] M. Iwata and S. Sasa, e-print arXiv:0808.0766

[23] M. Iwata and S. Sasa (unpublished) 